\title{
Unconscious Activation of the Prefrontal No-Go Network
}

\author{
Simon van Gaal, ${ }^{1,2}$ K. Richard Ridderinkhof, ${ }^{2}$ H. Steven Scholte, ${ }^{1}$ and Victor A. F. Lamme ${ }^{1}$ \\ ${ }^{1}$ Cognitive Neuroscience Group and ${ }^{2}$ Amsterdam Center for the Study of Adaptive Control in Brain and Behavior (Acacia), Department of Psychology, \\ University of Amsterdam, 1018 WB, Amsterdam, The Netherlands
}

Cognitive control processes involving prefrontal cortex allow humans to overrule and inhibit habitual responses to optimize performance in new and challenging situations, and traditional views hold that cognitive control is tightly linked with consciousness. We used functional magnetic resonance imaging to investigate to what extent unconscious "no-go" stimuli are capable of reaching cortical areas involved in inhibitory control, particularly the inferior frontal cortex (IFC) and the pre-supplementary motor area (pre-SMA). Participants performed a go/no-go task that included conscious (weakly masked) no-go trials, unconscious (strongly masked) no-go trials, as well as go trials. Replicating typical neuroimaging findings, response inhibition on conscious no-go stimuli was associated with a (mostly right-lateralized) frontoparietal "inhibition network." Here, we demonstrate, however, that an unconscious no-go stimulus also can activate prefrontal control networks, most prominently the IFC and the pre-SMA. Moreover, if it does so, it brings about a substantial slowdown in the speed of responding, as if participants attempted to inhibit their response but just failed to withhold it completely. Interestingly, overall activation in this "unconscious inhibition network" correlated positively with the amount of slowdown triggered by unconscious no-go stimuli. In addition, neural differences between conscious and unconscious control are revealed. These results expand our understanding of the limits and depths of unconscious information processing in the human brain and demonstrate that prefrontal cognitive control functions are not exclusively influenced by conscious information.

\section{Introduction}

Recent experiments revealed a plethora of often astounding effects of unconscious stimuli on behavior, perception, and cognition. To name a few, unconscious information has been shown to influence motivation (Pessiglione et al., 2007), reward value and decision making (Pessiglione et al., 2008), emotional processing (Whalen et al., 1998), object recognition (Stoerig and Cowey, 1997), semantic processing (Dehaene et al., 2001), and action planning/execution (Binsted et al., 2007). These thought-provoking results have raised important questions about the limits of unconscious cognition and the evolutionary function of consciousness (Dehaene and Naccache, 2001; Dehaene, 2008).

Although unconscious information seems to have a wide range of effects on many psychological mechanisms, a debatable case is the existence of unconscious cognitive control. Cognitive control becomes necessary when routine behavior (e.g., driving a car) is interrupted unexpectedly by information (e.g., a pedestrian crossing the street) that calls for behavioral adaptations (e.g., braking fiercely). Generally speaking, it is thought that one should be conscious of the control-initiating stimulus to implement control and to overcome or to inhibit automatized ongoing behavior ("to regain control") (Umilta, 1988; Jacoby, 1991; Merikle et al., 1995; Dehaene and Naccache, 2001; Jack and Shallice, 2001; Eimer and Schlaghecken, 2003). The recruitment and implemen-

Received June 23, 2009; revised Jan. 15, 2010; accepted Jan. 25, 2010.

This work is supported by an Advanced Investigator Grant from the European Research Council to V.A.F.L. and a Vici Grant from the Netherlands Organization of Scientific Research to K.R.R.

Correspondence should be addressed to Simon van Gaal, Department of Psychology, University of Amsterdam, Roetersstraat 15, 1018 WB, Amsterdam, The Netherlands. E-mail: s.vangaal@uva.nl.

DOI:10.1523/JNEUROSCI.2992-09.2010

Copyright $\odot 2010$ the authors $\quad 0270-6474 / 10 / 304143-08 \$ 15.00 / 0$ tation of such control processes depend strongly on the prefrontal cortex (PFC) (Fuster, 2000; Miller, 2000; Ridderinkhof et al., 2004), which is, of all brain regions, also the one most often associated with conscious experience (Rees et al., 2002; Crick and Koch, 2003; Kouider and Dehaene, 2007). Therefore, it seems likely that consciousness and cognitive control are intimately related and this belief is so strong that many authors naturally refer to the concept of "conscious cognitive control" as if "unconscious cognitive control" is inconceivable (for review, see Hommel, 2007; see also Suhler and Churchland, 2009). However, recently we (van Gaal et al., 2008, 2009) and others (Lau and Passingham, 2007) put this long-held assumption to a direct test and provided evidence for the existence of unconsciously triggered cognitive control.

To illustrate, in a recent electroencephalogram (EEG) study we demonstrated that a masked (unconscious) no-go stimulus can trigger inhibitory control (van Gaal et al., 2008), which commonly has been assumed to comprise conscious control mechanisms (Libet, 1999; Pisella et al., 2000; Eimer and Schlaghecken, 2003). Source imaging suggested that unconsciously triggered inhibitory control was associated with activity in the lateral PFC. In the present work, we tried to further illuminate the possible depth of processing of unconscious information using methods that allow more spatial precision in our neuroanatomical inferences, namely functional magnetic resonance imaging (fMRI). We devised a new go/no-go task in which conscious (weakly masked) and unconscious (strongly masked) no-go signals were randomly mixed with go signals. In this task, unconscious no-go signals triggered a substantial slowdown in the speed of responding, as if participants tried to cancel their response but just failed to inhibit it entirely. This allowed us to investigate to what extent 
unconscious no-go signals are capable of activating brain areas involved in (conscious) inhibitory control, such as the inferior frontal cortex (IFC) and the pre-supplementary motor area (preSMA) (Wager et al., 2005; Aron et al., 2007; Leung and Cai, 2007; Forstmann et al., 2008; Mostofsky and Simmonds, 2008; Chikazoe et al., 2009).

\section{Materials and Methods}

Participants. Twenty-nine undergraduate psychology students of the University of Amsterdam were recruited for a behavioral experiment $(1 \mathrm{~h})$ in which the task was explained and practiced. Twenty-four of these participants were selected to participate in the fMRI experiment (for selection methods, see below). All participants gave their written informed consent before participation, were right-handed as assessed by the Edinburgh inventory (Oldfield, 1971), had normal or corrected-to-normal vision, and were naive to the purpose of the experiments. All procedures were executed in compliance with relevant laws and institutional guidelines, and were approved by the local ethics committee.

The masked go/no-go task. The blood-oxygen level-dependent (BOLD) signal was measured while participants performed a newly devised go/ no-go task in which we randomly mixed weakly masked (conscious) and strongly masked (unconscious) no-go trials as well as go trials. In this task, participants were instructed to respond as fast as possible to a white annulus (the go signal, visual angle of $0.80^{\circ}$ ) but withhold their response when a white square (the no-go stimulus, visual angle of $0.47^{\circ} \times$ $0.47^{\circ}$ ) briefly preceded the annulus. However, when a diamond (i.e., the same square but tilted by $45^{\circ}$ ) preceded the annulus, they were instructed to respond as quickly as possible.

In one condition, the annulus was ineffective in masking the preceding stimulus (square/diamond) since the square/diamond was presented for a relatively long duration $(233 \mathrm{~ms})$ and the annulus for a fairly brief duration $(16.7 \mathrm{~ms})$. Therefore, the square/diamond was clearly visible. We will refer to this condition as the weakly masked condition. Crucially, in a second condition the square/diamond was presented very briefly $(16.7 \mathrm{~ms})$ and was followed after only a brief delay $(33 \mathrm{~ms})$ by the go annulus (duration $200 \mathrm{~ms}$ ). We will refer to these conditions as the strongly masked condition, because the annulus functioned as a metacontrast mask, which is known to strongly reduce stimulus visibility (Breitmeyer, 1984). The combination of these factors effectively rendered the participants incapable of perceiving the square/diamond, as evidenced by chance-level performance on a two-choice discrimination task administered after the experiment (see Results). In the strongly masked condition, participants just perceived a white annulus and therefore treated these trials as go trials.

Because the square functioned as a no-go signal in the weakly masked conditions, this stimulus was consistently associated with response inhibition. On the contrary, the diamond was not associated with response inhibition because participants were instructed to respond to trials containing a diamond. Because each of the four conditions (weakly/strongly masked $\times$ square/diamond) was presented in $25 \%$ of occasions, overall a weakly masked no-go signal was presented in $25 \%$ of the trials ( 30 trials of each condition; 120 in total per block). Because all conditions were presented equally frequently, we could investigate the processing of no-go signals without confounding response inhibition with the processing of infrequent stimuli (Chikazoe et al., 2009) (Fig. 1, stimulus and trial timing). The stimulus used as no-go signal (square or diamond) was counterbalanced across subjects. Note that from fixation cross-presentation, the duration until annulus offset of all conditions was equal (duration, $750 \mathrm{~ms}$ ).

Procedure. Before the actual fMRI experiment, participants were invited to the laboratory to practice the task $(1 \mathrm{~h})$. In a pilot study, participants reported seeing a "flicker" before the appearance of the annulus in the strongly masked conditions, as if the annulus quickly expanded when it appeared on the screen. Therefore, in the practice session before the actual fMRI experiment, participants were told that the white annulus quickly expanded (grew bigger) when it appeared on the screen. Most of the participants were able to follow the instructions and perform the task proficiently in the practice session already. However, in an informal in-

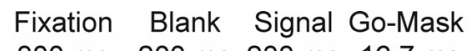
$300 \mathrm{~ms} 200 \mathrm{~ms} 233 \mathrm{~ms} 16.7 \mathrm{~ms}$

Weakly masked no-go trial $(25 \%)$
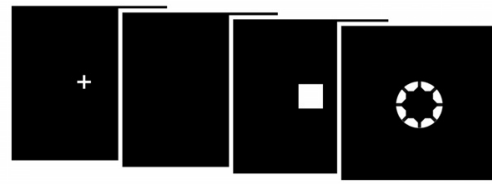

Weakly masked go trial $(25 \%)$
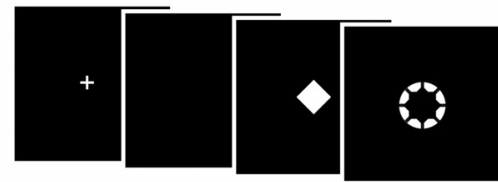

Fixation Blank Signal Blank Go-Mask $300 \mathrm{~ms} 200 \mathrm{~ms} 16.7 \mathrm{~ms} 33 \mathrm{~ms} 200 \mathrm{~ms}$

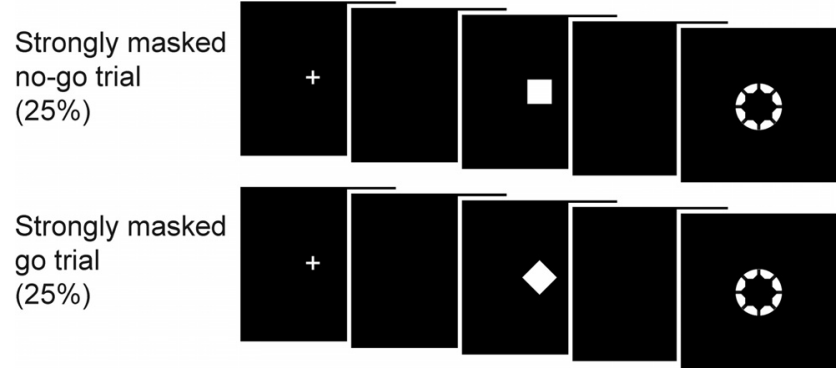

Figure 1. Experimental design. The duration of the square/diamond, the duration of the metacontrast go-annulus, and the stimulus onset asynchrony between the square/diamond and the metacontrast go-annulus were varied. To this end, the mask was unsuccessful in masking the preceding square/diamond on some occasions (weakly masked conditions) but rendered it invisible at others (strongly masked conditions; see Results). Thereby, two factors were manipulated [trial (go or no-go) and visibility (weakly masked or strongly masked)], which constitutes a $2 \times 2$ factorial design with the following four conditions: (1) a weakly masked no-go condition, (2) a weakly masked go condition, (3) a strongly masked no-go condition, and (4) a strongly masked go condition.

terview during the practice session, five participants indicated having difficulty differentiating between the conditions. Therefore, based on this observation, these participants were not invited to participate in the upcoming fMRI experiment.

During the scanning session, participants performed one block of the task outside the scanner. Inside the scanner, participants performed four blocks of the go/no-go task. Immediately after the imaging session, participants were informed about the fact that in the "annulus-only" condition an annulus actually was always preceded by a diamond or a square. Next, to test their ability to see these stimuli, participants performed a two-choice discrimination task while still lying in the scanner (48 trials; 24 trials of each strongly masked condition). Stimulus and trial timing was exactly the same as in the masked go/no-go task. After the presentation of a trial, a pair of choices was presented left and right of fixation. Participants were asked to determine whether a square or a diamond was presented in the preceding trial. The two alternatives remained on the screen until the subject made a response, after which a new trial started. Before performing this task, participants were told that squares and diamonds were presented equally frequently and were instructed to consider this in giving their response. They were also told that only accuracy was important in this task, not the speed of responding.

Behavioral data analyses. For the response time (RT) analyses, responses between 100 and 1000 ms were included. For the RT analysis, a paired two-tailed $t$ test was performed on mean RT on responded, strongly masked go trials and responded, strongly masked no-go trials. For the RT distributions, we calculated the number of responses in bins of $50 \mathrm{~ms}$ ranging from 100 to $700 \mathrm{~ms}$. Inhibition rates were computed by taking all trials without a response before the start of the next trial. For the analysis of the inhibition rates, a paired two-tailed $t$ test was performed on the square root of the percentage of responding on strongly masked go trials and strongly masked no-go trials. Discrimination performance was 
tested for significance for each individual participant using a binomial test evaluated at a $p$ value of 0.05 . Subsequently, on the group level, a one-sample $t$ test was performed on the $d$ scores (tested against 0 ).

fMRI scanning and analysis. Data were collected on a Philips 3T Intera scanner. The scanning session always started with a 3D T1 scan [T1 turbo field echo, $250 \mathrm{~mm}^{2}$ field of view (FOV), $256^{2}$ in-plane resolution, 182 slices, $1.2 \mathrm{~mm}$ slice thickness, $9.6 \mathrm{~s}$ repetition time (TR), $4.6 \mathrm{~ms}$ echo time (TE), $8^{\circ}$ flip angle (FA), sagittal orientation]. Next, four runs (lasting $\sim 8$ min each) of functional data were collected (2.29 s TR, $30 \mathrm{~ms}$ TE, 220 $\mathrm{mm}^{2} \mathrm{FOV}, 72^{2}$ in-plane resolution, 35 slices, $3.3 \mathrm{~mm}$ slice thickness, $90^{\circ}$ FA, transversal orientation) covering the whole brain. Each run contained 120 trials (30 of each condition). Trial sequences were optimized using OptSeq (http://surfer.nmr.mgh.harvard.edu/optseq). We used a rapid presentation design with an average trial time of $4 \mathrm{~s}$ (ranging from 2 to $16 \mathrm{~s}$ ). Stimuli were presented on a back-projection screen, which was viewed via a mirror system attached to the MRI headcoil.

FEAT (FMRI Expert Analysis Tool) version 4.1, part of FSL [Oxford Centre for Functional MRI of the Brain (FMRIB) Software Library (www.fmrib.ox.ac.uk/fsl)] was used to analyze the fMRI data. fMRI images were realigned to compensate for small head movements (Jenkinson et al., 2002). Functional data were aligned to the structural image of the subject, and the data of each subject were transformed to the standard space of the MNI (Montreal Neurological Institute) using FLIRT. Next, the functional data were spatially smoothed using a $5 \mathrm{~mm}$ full-width-athalf-maximum Gaussian kernel and high-pass filtered in the temporal domain $(\sigma=40 \mathrm{~s})$. Finally, the functional data were prewhitened using FSL (Woolrich et al., 2001).

The following conditions were separately modeled by convolution with a double-gamma response function in a general linear model: (1) inhibited, weakly masked no-go trials; (2) responded, weakly masked no-go trials; (3) responded, weakly masked go trials; (4) responded, strongly masked no-go trials; and (5) responded, strongly masked go trials. The theoretically possible other trial types (e.g., nonresponded, weakly masked go trials; nonresponded, strongly masked go trials; or nonresponded, strongly masked no-go trials) were not encountered often enough in all subjects to warrant inclusion. Runs were pooled on a per subject basis using a fixed-effects model. Subsequently, a mixedeffects group analysis was conducted using the FMRIB FLAME stages 1 and 2 , in which relevant lower-level contrasts were combined. Two participants did not have any trials to model the condition in which weakly masked no-go trials were responded to, in one of the four runs. These two runs were excluded from all analyses.

First, we identified voxels involved in consciously triggered response inhibition by contrasting inhibited, weakly masked no-go trials with responded, weakly masked go trials. For this contrast, we report cortical regions with a height threshold of $Z>2.3$ and a cluster probability of $p<$ 0.05 , thereby correcting for whole-brain multiple comparisons [using Gaussian random field theory (GRFT)] (Worsley, 2001). Next, we looked for significant activations related to unconsciously triggered inhibitory control by contrasting responded, strongly masked no-go trials with responded, strongly masked go trials. We report regions about which we had an a priori hypothesis (IFC and pre-SMA) with a height threshold of $Z>2.3$ and a cluster probability of $p<0.05$, using a correction for multiple comparisons (GRFT) across a small volume of interest (Worsley et al., 1996). Volumes of interest were taken from the Harvard-Oxford Cortical Structural Atlas that accompanies FSL. For the IFC, we used a mask of the bilateral frontal operculum cortex ( 4204 voxels, $34 \mathrm{~cm}^{3}$ ), and for the pre-SMA, a mask of the juxtapositional lobule cortex (5970 voxels, $48 \mathrm{~cm}^{3}$ ). Both anatomical regions include the peaks of inhibitionrelated activation observed in many previous no-go and stop-signal studies (Bellgrove et al., 2004; Wager et al., 2005; Aron and Poldrack, 2006; Blasi et al., 2006; Leung and Cai, 2007; Forstmann et al., 2008; Simmonds et al., 2008; Xue et al., 2008; Duann et al., 2009). We would like to note that no regions survived multiple-comparison corrections when we narrowed our search space to the conscious inhibition activations. This might be due to the fact that the conscious mask was relatively large $\left(13,411\right.$ voxels, $\left.107 \mathrm{~cm}^{3}\right)$ and because the consciously and unconsciously activated clusters were not perfectly overlapping (see Results).
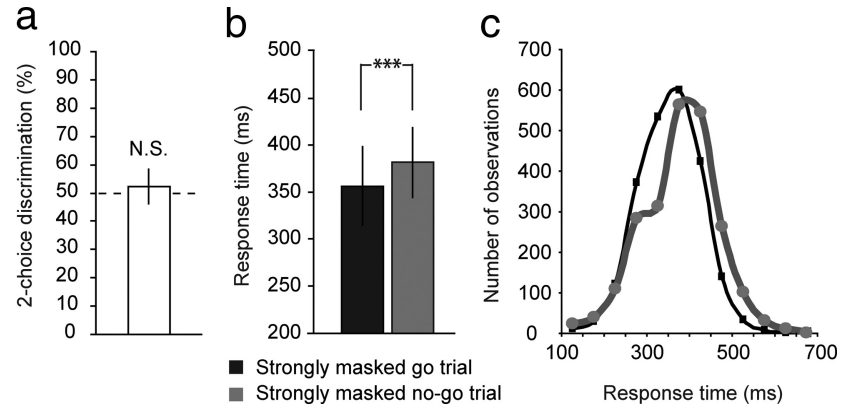

Figure 2. Strongly masked (unconscious) no-go signals slow down responses. $\boldsymbol{a}$, Participants were unable to discriminate between trials with a strongly masked square or diamond, as revealed by chance-level performance in a two-choice discrimination task administered after the main experiment. $\boldsymbol{b}$, Although strongly masked no-go signals could not be perceived consciously, they still triggered inhibitory control processes, as revealed by significantly longer response times on these trials than on strongly masked go trials. c, Response time distributions of strongly masked no-go trials and strongly masked go trials. All plots indicate mean performance \pm intersubject SDs.

To visualize the temporal profile of the BOLD response in the right IFC for each of the four conditions, we selected all overlapping right IFC voxels from the conscious contrast and the unconscious contrast, and performed a deconvolution analysis. For the deconvolution analysis, we up-sampled the temporal resolution of the BOLD signal to $1.14 \mathrm{~s}(\mathrm{TR} / 2)$ and modeled the BOLD response in the period of -3 to $16 \mathrm{~s}$ per run, per subject with slightly smoothed delta functions that peaked each $1.14 \mathrm{~s}$ (Dale and Buckner, 1997). These were subsequently averaged over runs and subjects (see Fig. $4 c$ ).

For all correlational analyses, we performed nonparametric Spearman's rank correlations evaluated at $\alpha=0.05$ (two-tailed).

\section{Results}

\section{Probing the effectiveness of the masking procedure}

A prerequisite for studying the neural mechanisms of unconsciously triggered inhibitory control is the effectiveness of our masking procedure. To assess whether participants were truly unaware of strongly masked squares and diamonds, a two-choice discrimination task was administered after the go/no-go task while participants were still lying in the scanner. The discrimination task was administered after the main experiment to rule out any effect of perceptual learning during the experiment. In this conservative measure of stimulus visibility, 20 of 24 participants scored at chance level (binomial test, $p>0.05$ ), suggesting that these individuals were unable to perceive strongly masked squares/diamonds. Because we cannot ascertain that the other four participants were truly unaware of strongly masked signals during the go/no-go experiment, these participants were excluded from all further analyses. Also at the group level, discrimination performance $\left(d^{\prime}\right)$ did not deviate from chance level for the included 20 participants $\left(d^{\prime}=0.118, \mathrm{SD}=0.32\right.$, mean percentage correct $=52.3 \%, \mathrm{SD}=6.15, t_{(19)}=1.667, p=0.112$ ) (Fig. 2a). After the presentation of the behavioral and neuroimaging results, several additional correlational analyses between behavioral/brain data and discrimination scores will be reported that further indicate that participants were unable to perceive strongly masked squares/diamonds in the present experiment.

\section{Strongly masked (unconscious) no-go signals slow down responses}

Participants were able to perform this go/no-go task proficiently, as evidenced by typical inhibition rates of $69.9 \%$ on weakly masked no-go trials, while still responding quickly to weakly masked go trials (367 ms). Whereas weakly masked no-go trials 
triggered complete response inhibition in the majority of trials, participants did not inhibit their responses more often in strongly masked no-go trials $(0.58 \%)$ than in strongly masked go trials $(0.46 \%)$. Crucially, however, RTs were significantly longer to strongly masked no-go trials than to strongly masked go trials $\left(t_{(19)}=6.24 ; p<0.001\right.$ ) (Fig. $2 b$ ). Moreover, the entire response time distribution of strongly masked no-go trials was shifted in time compared with the response time distribution of strongly masked go trials (Fig. 2c), which indicates that RT slowing induced by strongly masked no-go signals was not due to only a few trials. The combination of these results indicates that strongly masked no-go signals triggered inhibitory control processes, but not sufficiently to withhold the overt response. Although not successful as such, the attempt to inhibit may have resulted in a slower buildup of response activation, thus leading to slowing of the imminent response.

\section{Neural mechanisms of conscious and unconscious inhibitory control}

To examine the neural mechanisms underlying consciously triggered inhibitory control, we contrasted inhibited, weakly masked no-go trials with responded, weakly masked go trials $(Z>2.3$, whole-brain cluster corrected). Consciously initiated response inhibition was associated with a typical (mostly right-lateralized) frontoparietal network associated with no-go inhibition (four large clusters; for a full list of activations see supplemental Table 1, available at www.jneurosci.org as supplemental material). This "conscious inhibition network" included the right and left IFC bordering and extending into the anterior insula, the pre-SMA, the anterior cingulate cortex, the right superior frontal gyrus, the right dorsolateral PFC, the right middle frontal gyrus, and bilateral inferior and superior parietal cortices (Fig. 3). This network is consistent with previous results of (conscious) response inhibition, in which the IFC and pre-SMA have been proposed to play important roles (Konishi et al., 1999; Garavan et al., 2002; Rubia et al., 2003; Wager et al., 2005; Blasi et al., 2006; Aron et al., 2007; Chikazoe et al., 2007; Simmonds et al., 2008; Duann et al., 2009).

To examine the activation related to the unconscious initiation of inhibitory control, we contrasted responded, strongly masked no-go trials with responded, strongly masked go trials. Significant activations were observed in three a priori hypothesized brain regions: the right IFC $(36,28,2 ; Z=4.10)$, the left IFC $(-42,12$, $-4 ; Z=3.71)$, and the pre-SMA $(-6,2$, $52 ; Z=3.52)$, corrected for multiple comparisons across a small volume of interest (Fig. 4a). Although these three brain regions were observed at approximately similar locations as the conscious activations, there were some small regional differences. Whereas the conscious and unconscious contrasts revealed a highly similar cluster in the right IFC (172 of 187 unconsciously activated voxels overlapped with consciously activated voxels, 92\% overlap), the left IFC (25 of 184 overlapping voxels, $14 \%$ overlap) and the pre-SMA (21 of 317 overlapping voxels, $7 \%$ overlap) overlapped less. Especially the
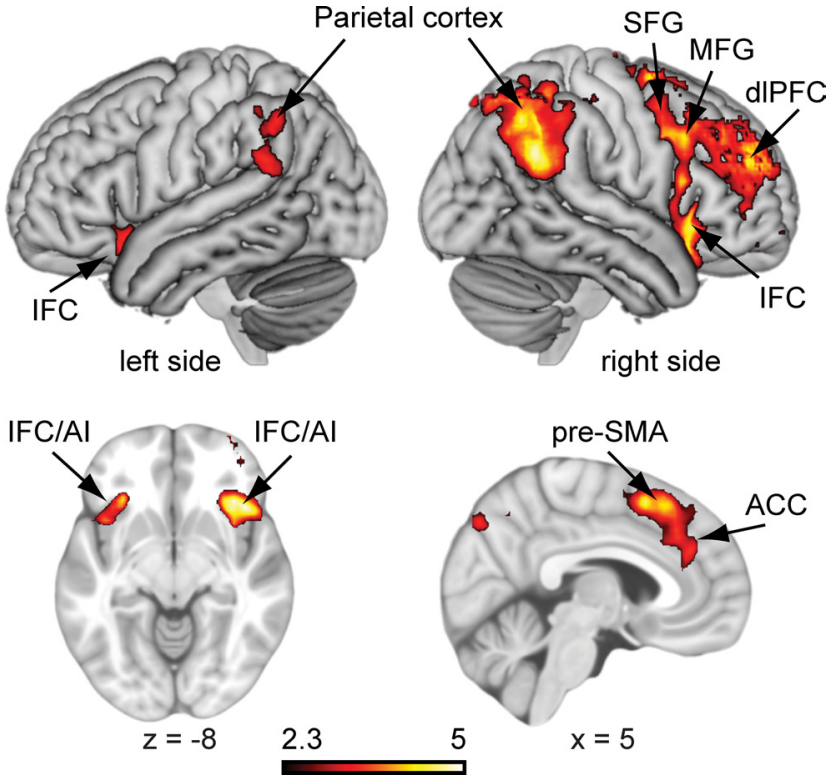

Figure 3. Neural activation associated with consciously triggered no-go inhibition. The contrast between inhibited, weakly masked no-go trials and responded, weakly masked go trials revealed activation in a (largely right-lateralized) frontoparietal inhibition network. For a full list of activated regions ( $Z>2.3$, whole-brain cluster corrected, $p<0.05$, GRFT), see supplemental Table 1 (available at www.jneurosci.org as supplemental material). ACC, Anterior cingulate cortex; Al, anterior insula; dIPFC, dorsolateral prefrontal cortex; MFG, middle frontal gyrus; SFG, superior frontal gyrus. a

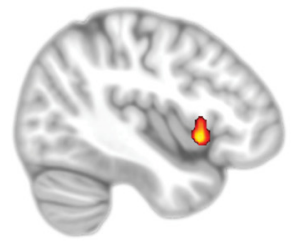

$x=-42$

b

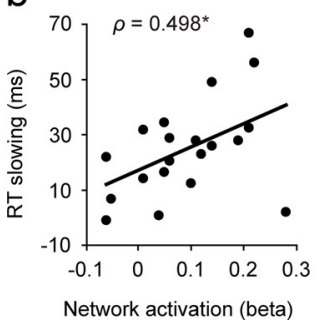

pre-SMA

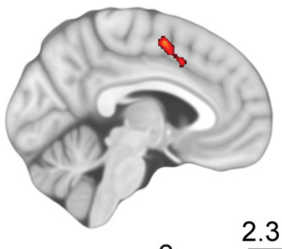

2.3

$x=-2$ right IFC

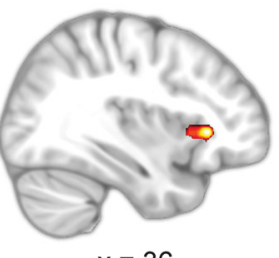

$x=36$
C Strongly masked conditions

Weakly masked conditions
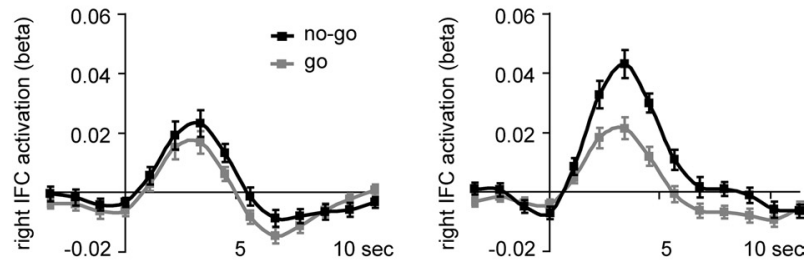

Figure 4. Neural activation associated with unconsciously triggered no-go inhibition. $\boldsymbol{a}$, The contrast between responded, strongly masked no-go trials and responded, strongly masked go trials revealed significant activation in three a priori hypothesized regions of interest (pre-SMA and left/right IFC), corrected for multiple comparisons ( $p<0.05, \mathrm{GRFT}$ ) across a small volume of interest (see Materials and Methods). $\boldsymbol{b}$, Across-subjects Spearman's rank correlation ( $\rho=0.498, p=0.026$ ) between unconsciously initiated RT slowing and activation in the unconscious inhibition network (the mean activation of all three clusters). c, BOLD time courses for all four conditions in the right IFC. The left panel depicts the strongly masked conditions, and the right panel depicts the weakly masked conditions. Scale bars, \pm intersubject SEMs.

part of the pre-SMA that was activated unconsciously was observed more posteriorly and ventrally compared with when it was activated consciously (Figs. 3, 4).

To test whether this unconsciously initiated activation pattern is truly related to the initiation of inhibitory control, we further examined whether individual differences in activation levels in the unconscious inhibition network could explain why strongly 
masked no-go signals slow down responses for some participants more than for others. More specifically, we extracted the data of each subject from each of the three activated regions and subsequently averaged these values across the three regions. Next, this mean activation measure was correlated with the amount of slowdown triggered by strongly masked no-go signals (mean RT strongly masked no-go trials $>$ mean RT strongly masked go trials) across all participants. Note that both measures were derived from completely independent analyses. Interestingly, overall activation in the "unconscious inhibition network" correlated positively with the amount of slowdown $(\rho=0.498, p=0.026)$ (Fig. 4b). Thus, the strength of activation in the unconscious inhibition network predicted the extent to which individuals slow down their responses to strongly masked no-go signals, which further supports that the observed unconscious activations are "functional" in the sense that they predict the impact of strongly masked no-go signals on subsequent behavior.

As a next step, we investigated whether this positive correlation between brain activation and behavioral performance was driven by activation in certain specific clusters within the unconscious inhibition network. Therefore, post hoc correlational analyses between brain activation and RT slowing were performed on each individual cluster. Significant positive correlations were observed in the right IFC $(\rho=0.465, p=0.039)$ and the left IFC $(\rho=0.509, p=0.022)$, but not in the pre-SMA $(\rho=0.188, p=$ 0.427 ). Thus, activation in the right IFC and left IFC predicted the extent to which individuals slowed down their responses after the presentation of a strongly masked no-go signal, suggesting an important role of these areas in processing strongly masked no-go signals and the subsequent implementation of response inhibition. For illustration purposes, we show the BOLD time courses for all four conditions in the right IFC, the cluster with the largest overlap across conscious and unconscious response inhibition (Fig. 4c).

The next set of analyses was performed for exploratory purposes. First, at a whole-brain level, we checked for unconsciously activated brain regions significant at $p<0.001$ uncorrected $(Z>$ 3.1 , extent threshold of five contiguous voxels) to explore the possibility that we missed strongly activated clusters outside the a priori expected regions of interest. First of all, the right IFC, the left IFC, and the pre-SMA were the clusters that survived this threshold most prominently (supplemental Table 2, available at www.jneurosci.org as supplemental material), which confirms our a priori hypothesis about the important role of these structures in unconscious (as well as conscious) inhibitory control. Further, besides the IFC and the pre-SMA, at this threshold some other clusters were also observed (including the posterior cingulate cortex, the frontal pole, and the superior parietal lobule), some of which were also observed in the conscious contrast at the same threshold (supplemental Table 1, available at www.jneurosci.org as supplemental material) as well as in many previous experiments (Durston et al., 2002; Wager et al., 2005; Aron and Poldrack, 2006; Li et al., 2006; Aron et al., 2007; Boehler et al., 2008; Braet et al., 2009). Follow-up experiments need to be performed to test the reliability of these latter effects.

Further, we explored the contribution of subcortical structures in the present experimental design. Various previous studies have demonstrated a key role of some basal ganglia structures, including the striatum (Casey et al., 1997; Garavan et al., 2002; Wager et al., 2005) and the subthalamic nucleus (Kühn et al., 2004; Aron and Poldrack, 2006; van den Wildenberg et al., 2006; Isoda and Hikosaka, 2008) in response inhibition. Here, at the thresholds used, no activation in subcortical structures sur- vived for either the conscious contrast (weakly masked no-go $>$ weakly masked go) or the unconscious contrast (strongly masked no-go $>$ strongly masked go). To explore the role of basal ganglia structures in conscious and unconscious response inhibition, we created anatomically based regions of interest (ROIs) [left/right putamen, left/right caudate, left/right pallidum, left/right accumbens, left/right thalamus (taken from the Harvard-Oxford Subcortical Structural Atlas)], extracted the activation level of each subject from each ROI, and tested for each ROI whether weakly masked no-go trials differed from weakly masked go trials. The same was done for the strongly masked conditions. These analyses did not yield any significant results (two-tailed $t$ tests evaluated at $p<0.05$, corrected for multiple comparisons).

Although all 20 of the included participants were unable to perceive strongly masked squares/diamonds consciously, as illustrated by chance performance on a two-choice discrimination task performed after the go/no-go experiment, we sought to further rule out the possibility that the reported behavioral or neuroimaging results can be explained by accidental visibility of masked stimuli. If incidental no-go visibility were responsible for the observed effects, one would expect reliable positive correlations between discrimination scores $\left(d^{\prime}\right)$ and neuroimaging/behavioral correlates of inhibition. However, this was not the case for mean activation in the unconscious inhibition network (IFC and pre-SMA together: $\rho=-0.113, p=0.634$ ) or activation in any of the three clusters separately (largest $\rho=0.028$ ). Discrimination performance also did not correlate significantly with unconsciously triggered RT slowing ( $\rho=0.343, p=0.139$ ). Crucially, RT slowing effects were still significant when discrimination performance was extrapolated to zero visibility. The linear regression analysis revealed a significant intercept $(21.9 \mathrm{~ms}$; $p<0.001$, for the regression of RT slowing against $\left.d^{\prime}\right)$, which further confirms the conclusion that RT slowing was induced by no-go signals that could not be perceived consciously [see Greenwald et al. (1995) and Hannula et al. (2005) for further discussion and justification of this method]. Although one should be cautious in claiming effects being caused by unconscious stimuli, the absence of consistent positive correlations between discrimination performance and brain/behavioral measures suggests that the reported effects are not related to (accidental) visibility of strongly masked stimuli.

\section{Discussion}

In a go/no-go paradigm, we masked no-go signals to the point that they could no longer be detected to investigate the depth of processing of strongly masked (unconscious) no-go signals in the human brain. Strongly masked no-go signals were observed to activate brain regions central to networks that have been associated with conscious response inhibition, namely the IFC and the pre-SMA (Konishi et al., 1999; Wager et al., 2005; Aron and Poldrack, 2006; Blasi et al., 2006; Chambers et al., 2006; Chikazoe et al., 2007; Leung and Cai, 2007; Forstmann et al., 2008; Mostofsky and Simmonds, 2008; Simmonds et al., 2008). Activation in this "unconscious inhibition network" appeared to be functional, in that this activation correlated with the amount of slowdown of manual responses elicited by strongly masked no-go signals, as if participants attempted to suppress their response but just failed to inhibit it fully. These results demonstrate that inhibitory control functions of the PFC can be triggered unconsciously and thereby extend traditional views that tightly link cognitive control to consciousness (Umilta, 1988; Jacoby, 1991; Merikle et al., 1995; Dehaene and Naccache, 2001; Eimer and Schlaghecken, 
2003; Hommel, 2007; Suhler and Churchland, 2009). Further, these results expand the proposed depth of processing of unconscious stimuli in the human brain (Rees et al., 2002; Crick and Koch, 2003; Kouider and Dehaene, 2007).

Interestingly, the correlation between brain activation and RT slowing triggered by strongly masked no-go signals was driven mainly by activation in the bilateral IFC, suggesting an important role of this area in implementing inhibitory control unconsciously. Although most attention is devoted to the right IFC in response inhibition, generally, both hemispheres are activated when participants inhibit their response in the go/no-go task as well as the stop-signal task; however, as also observed here, the right was activated slightly more than the left (Konishi et al., 1999; Wager et al., 2005; Aron and Poldrack, 2006; Blasi et al., 2006; Aron et al., 2007; Chikazoe et al., 2007, 2009; Leung and Cai, 2007; Xue et al., 2008). IFC activation (during conscious and unconscious no-go inhibition) is interesting since we included an even number of go and no-go trials, which renders the attribution of activation in this area to "oddball" related effects [due to the infrequency of no-go trials, as has been suggested recently (Mostofsky and Simmonds, 2008; Duann et al., 2009)] less likely. Whether the IFC is truly crucial for unconscious inhibitory control should be verified in the future, e.g., by transcranial magnetic stimulation or lesion studies.

Because participants practiced the task before going into the scanner, no-go signals were strongly associated with response inhibition during scanning. Thus, from this experiment, we can only conclude that inhibitory control-related brain areas can be activated unconsciously in situations in which a controlinitiating stimulus is consistently (and frequently) paired with inhibiting a motor response. In a recent behavioral study, we have shown that practice indeed plays an important role in the impact that masked (unconscious) stop signals have on behavior; RT slowing increased with task training across three sessions and was even absent in the first session (van Gaal et al., 2009). However, when we used the word "stop" (highly trained stimulus material) as a stop signal, unconscious RT slowing already was present in the first session. Interestingly, others have recently shown that practice on a more traditional go/no-go and stop task can indeed lead to the development of an automatic bottom-up form of inhibitory control due to the increased strength of stimulus-stop mappings (Verbruggen and Logan, 2008). Overall, it seems that inhibitory control benefits from frequent exposure to stimuli that are associated with response inhibition and that unconscious control needs more training to develop than conscious control (see also Dehaene, 2008).

These results fit nicely with the present findings and suggest that unconscious inhibitory control (as studied here) can be considered as a relatively "bottom-up" form of cognitive control. This is in line with recent studies demonstrating that unconscious stimuli can be processed in a fast-feedforward (bottom-up) manner, but do not seem to elicit recurrent (top-down) interactions between higher- and lower-level brain areas that allow information to be broadcasted across the brain and maintained across time (Dehaene et al., 2006; Lamme, 2006). Here, weakly masked (conscious) no-go signals elicited a typical large-scale frontoparietal inhibition network, and seemingly, strongly masked (unconscious) no-go stimuli can travel along similar processing routes, even up to prefrontal cortex. However, if they do so, they do not activate a similarly strong, stable, and extended activation pattern as do conscious stimuli (see also the BOLD results) but only cause a "trickle of activation" in specialized, but relatively isolated, no-go processors "that modulates decision time but does not determine the decision outcome" (Dehaene, 2008).

The present findings converge with and extend recent EEG results, obtained using a similar masked go/no-go paradigm, in important ways (van Gaal et al., 2008). In that EEG study, strongly masked (unconscious) no-go signals elicited a medium latency (from $\sim 300 \mathrm{~ms}$ ) frontal event-related potential (ERP) component that correlated with the extent to which participants slowed down responses to those no-go signals. Source imaging suggested that this frontal ERP component was localized in the lateral PFC. The present fMRI results specify these findings and pinpoint the IFC (and possibly the pre-SMA) as important components in linking strongly masked no-go signals to appropriate action, in this case attempting to withhold the overt response. Further, in the present fMRI experiment participants slowed down their responses much more than in the EEG study, which might be due to the fact that, in the present experiment, conditions were nicely balanced with respect to low-level stimulus properties, whereas this was less the case for the EEG experiment. Interestingly, RT distributions of strongly masked no-go trials and strongly masked go trials (Fig. $2 c$ ) started to differentiate after $\sim 250-300 \mathrm{~ms}$ (this is not a precise measure), which approximately corresponds to the latency range of the N2/P3 components, which are thought to be related to the conscious (Bokura et al., 2001; Schmajuk et al., 2006) and unconscious (van Gaal et al., 2008) activation of inhibitory control in stop-signal as well as go/no-go tasks (these ERP components tend to peak from 200 $\mathrm{ms}$ after stimulus presentation onward). We verified that our sample did not consist of clearly separated fast and slow subgroups, such that the present pattern could not be attributed to artifacts resulting from averaging across such subgroups. Future studies need to be performed to unravel the exact timing of the unconscious activation of inhibitory control in the prefrontal cortex.

In real life, unconscious cognitive control processes may come in very handy. Over the course of a day, we are overwhelmed by a constant stream of stimuli entering our brains, some of which may reach conscious awareness and some of which probably do not. Several lines of evidence suggest that the prefrontal cortex plays a top-down biasing role (Miller and Cohen, 2001) in inhibiting many of the unconsciously activated response tendencies associated with these stimuli (Sumner et al., 2007; Sumner and Husain, 2008). Although some of these might be beneficial, many others probably are not. The (unconscious) suppression of most of these action tendencies probably helps us to avoid many embarrassing or socially inappropriate situations and might be crucial for adaptive goal-directed behavior (Suhler and Churchland, 2009).

A remaining outstanding question concerns why we were able to provide evidence for prefrontal processing of unconscious information, whereas many other studies were not. It has been observed that the strength of unconscious activation decays rapidly during its way up in the cortical hierarchy (Grill-Spector et al., 2000; Dehaene et al., 2001); therefore, the effects of masked stimuli at higher-level cortical areas have generally been observed to be small and not to include the prefrontal cortex (for review, see Dehaene and Naccache, 2001; Kouider and Dehaene, 2007; but see Lau and Passingham, 2007; Thompson and Schall, 1999; van Gaal et al., 2008). We hypothesize that we were able to provide evidence for unconscious prefrontal cognitive control because our approach differs fundamentally from previous masking studies, as it combines two factors that, to our knowledge, rarely have been combined before in neuroimaging studies. First, in the 
present experiment, the unconscious stimulus of interest is highly task relevant and attended. This is important, since it has been shown that attended and task-relevant stimuli are processed faster and more deeply in the human brain than unattended and taskirrelevant information (for reviews, see Kanwisher and Wojciulik, 2000; Kastner and Ungerleider, 2000; Lamme and Roelfsema, 2000). Interestingly, recent studies demonstrated that attention can also be oriented toward unconscious stimuli, which subsequently enhances the influence of these stimuli on behavior (Naccache et al., 2002; Sumner et al., 2006).

Second, and perhaps more importantly, in our study the unconscious stimulus of interest was strongly associated with prefrontal cognitive control functions. Therefore, the instructed task set of the participant required a deep level of information processing (incorporating prefrontal cortex) of the unconscious stimulus. Recently, we (van Gaal et al., 2008) and others (Nakamura et al., 2006, 2007) have shown that the instructed top-down task set strongly determines the processing routes taken by masked stimuli. The combination of both factors allowed us to tap more directly into the possible scope and depth of unconscious information processing than previous studies using the masking task (or related paradigms). Although we have yet to understand the exact functional and neural differences between consciously and unconsciously triggered cognitive control, these results stretch the alleged limits and depth of unconscious information processing in the human brain and directly impact the current debate about the proposed tight relationship between consciousness and cognitive control.

\section{References}

Aron AR, Poldrack RA (2006) Cortical and subcortical contributions to stop signal response inhibition: role of the subthalamic nucleus. J Neurosci 26:2424-2433.

Aron AR, Behrens TE, Smith S, Frank MJ, Poldrack RA (2007) Triangulating a cognitive control network using diffusion-weighted magnetic resonance imaging (MRI) and functional MRI. J Neurosci 27:3743-3752.

Bellgrove MA, Hester R, Garavan H (2004) The functional neuroanatomical correlates of response variability: evidence from a response inhibition task. Neuropsychologia 42:1910-1916.

Binsted G, Brownell K, Vorontsova Z, Heath M, Saucier D (2007) Visuomotor system uses target features unavailable to conscious awareness. Proc Natl Acad Sci U S A 104:12669-12672.

Blasi G, Goldberg TE, Weickert T, Das S, Kohn P, Zoltick B, Bertolino A, Callicott JH, Weinberger DR, Mattay VS (2006) Brain regions underlying response inhibition and interference monitoring and suppression. Eur J Neurosci 23:1658-1664.

Boehler CN, Münte TF, Krebs RM, Heinze HJ, Schoenfeld MA, Hopf JM (2009) Sensory MEG responses predict successful and failed inhibition in a stop-signal task. Cereb Cortex 19:134-145.

Bokura H, Yamaguchi S, Kobayashi S (2001) Electrophysiological correlates for response inhibition in a go/no-go task. Clin Neurophysiol 112:2224-2232.

Braet W, Johnson KA, Tobin CT, Acheson R, Bellgrove MA, Robertson IH, Garavan H (2009) Functional developmental changes underlying response inhibition and error-detection processes. Neuropsychologia 47:3143-3151.

Breitmeyer BG (1984) Visual masking: an integrative approach. Oxford: Oxford UP.

Casey BJ, Castellanos FX, Giedd JN, Marsh WL, Hamburger SD, Schubert AB, Vauss YC, Vaituzis AC, Dickstein DP, Sarfatti SE, Rapoport JL (1997) Implication of right frontostriatal circuitry in response inhibition and attention-deficit/hyperactivity disorder. J Am Acad Child Adolesc Psychiatry $36: 374-383$.

Chambers CD, Bellgrove MA, Stokes MG, Henderson TR, Garavan H, Robertson IH, Morris AP, Mattingley JB (2006) Executive "brake failure" following deactivation of human frontal lobe. J Cogn Neurosci 18:444-455.

Chikazoe J, Konishi S, Asari T, Jimura K, Miyashita Y (2007) Activation of right inferior frontal gyrus during response inhibition across response modalities. J Cogn Neurosci 19:69-80.

Chikazoe J, Jimura K, Asari T, Yamashita K, Morimoto H, Hirose S, Miyashita Y, Konishi S (2009) Functional dissociation in right inferior frontal cortex during performance of go/no-go task. Cereb Cortex 19:146-152.

Crick F, Koch C (2003) A framework for consciousness. Nat Neurosci 6:119-126.

Dale AM, Buckner RL (1997) Selective averaging of rapidly presented individual trials using fMRI. Hum Brain Mapp 5:329-340.

Dehaene S (2008) Conscious and nonconscious processes: distinct forms of evidence accumulation? In: Decision making, the human mind, and implications for institutions. Strüngmann forum reports (Engel C, Singer W, eds), pp 21-49. Cambridge, MA: MIT.

Dehaene S, Naccache L (2001) Towards a cognitive neuroscience of consciousness: basic evidence and a workspace framework. Cognition 79:1-37.

Dehaene S, Naccache L, Cohen L, Bihan DL, Mangin JF, Poline JB, Rivière D (2001) Cerebral mechanisms of word masking and unconscious repetition priming. Nat Neurosci 4:752-758.

Dehaene S, Changeux JP, Naccache L, Sackur J, Sergent C (2006) Conscious, preconscious, and subliminal processing: a testable taxonomy. Trends Cogn Sci 10:204-211.

Duann JR, Ide JS, Luo X, Li CS (2009) Functional connectivity delineates distinct roles of the inferior frontal cortex and presupplementary motor area in stop signal inhibition. J Neurosci 29:10171-10179.

Durston S, Thomas KM, Worden MS, Yang Y, Casey BJ (2002) The effect of preceding context on inhibition: an event-related fMRI study. Neuroimage 16:449-453.

Eimer M, Schlaghecken F (2003) Response facilitation and inhibition in subliminal priming. Biol Psychol 64:7-26.

Forstmann BU, van den Wildenberg WP, Ridderinkhof KR (2008) Neural mechanisms, temporal dynamics, and individual differences in interference control. J Cogn Neurosci 20:1854-1865.

Fuster JM (2000) Executive frontal functions. Exp Brain Res 133:66-70.

Garavan H, Ross TJ, Murphy K, Roche RA, Stein EA (2002) Dissociable executive functions in the dynamic control of behavior: inhibition, error detection, and correction. Neuroimage 17:1820-1829.

Greenwald AG, Klinger MR, Schuh ES (1995) Activation by marginally perceptible (subliminal) stimuli: dissociation of unconscious from conscious cognition. J Exp Psychol Gen 124:22-42.

Grill-Spector K, Kushnir T, Hendler T, Malach R (2000) The dynamics of object-selective activation correlate with recognition performance in humans. Nat Neurosci 3:837-843.

Hannula DE, Simons DJ, Cohen NJ (2005) Imaging implicit perception: promise and pitfalls. Nat Rev Neurosci 6:247-255.

Hommel B (2007) Consciousness and control: not identical twins. J Consciousness Stud 14:155-167.

Isoda M, Hikosaka O (2008) Role for subthalamic nucleus neurons in switching from automatic to controlled eye movement. J Neurosci 28:7209-7218.

Jack AI, Shallice T (2001) Introspective physicalism as an approach to the science of consciousness. Cognition 79:161-196.

Jacoby LL (1991) A process dissociation framework: separating automatic from intentional uses of memory. J Mem Lang 30:513-541.

Jenkinson M, Bannister P, Brady M, Smith S (2002) Improved optimization for the robust and accurate linear registration and motion correction of brain images. Neuroimage 17:825-841.

Kanwisher N, Wojciulik E (2000) Visual attention: insights from brain imaging. Nat Rev Neurosci 1:91-100.

Kastner S, Ungerleider LG (2000) Mechanisms of visual attention in the human cortex. Annu Rev Neurosci 23:315-341.

Konishi S, Nakajima K, Uchida I, Kikyo H, Kameyama M, Miyashita Y (1999) Common inhibitory mechanism in human inferior prefrontal cortex revealed by event-related functional MRI. Brain 122:981-991.

Kouider S, Dehaene S (2007) Levels of processing during non-conscious perception: a critical review of visual masking. Philos T R Soc B 362: $857-875$.

Kühn AA, Williams D, Kupsch A, Limousin P, Hariz M, Schneider GH, Yarrow K, Brown P (2004) Event-related beta desynchronization in human subthalamic nucleus correlates with motor performance. Brain 127:735-746.

Lamme VA (2006) Towards a true neural stance on consciousness. Trends Cogn Sci 10:494-501. 
Lamme VA, Roelfsema PR (2000) The distinct modes of vision offered by feedforward and recurrent processing. Trends Neurosci 23:571-579.

Lau HC, Passingham RE (2007) Unconscious activation of the cognitive control system in the human prefrontal cortex. J Neurosci 27:5805-5811.

Leung HC, Cai W (2007) Common and differential ventrolateral prefrontal activity during inhibition of hand and eye movements. J Neurosci 27:9893-9900.

Li CS, Huang C, Constable RT, Sinha R (2006) Imaging response inhibition in a stop-signal task: neural correlates independent of signal monitoring and post-response processing. J Neurosci 26:186-192.

Libet B (1999) Do we have free will? J Consciousness Stud 12:47-57.

Merikle PM, Joordens S, Stolz JA (1995) Measuring the relative magnitude of unconscious influences. Conscious Cogn 4:422-439.

Miller EK (2000) The prefrontal cortex and cognitive control. Nat Rev Neurosci 1:59-65.

Miller EK, Cohen JD (2001) An integrative theory of prefrontal cortex function. Annu Rev Neurosci 24:167-202.

Mostofsky SH, Simmonds DJ (2008) Response inhibition and response selection: two sides of the same coin. J Cogn Neurosci 20:751-761.

Naccache L, Blandin E, Dehaene S (2002) Unconscious masked priming depends on temporal attention. Psychol Sci 13:416-424.

Nakamura K, Hara N, Kouider S, Takayama Y, Hanajima R, Sakai K, Ugawa Y (2006) Task-guided selection of the dual neural pathways for reading. Neuron 52:557-564.

Nakamura K, Dehaene S, Jobert A, Le Bihan D, Kouider S (2007) Taskspecific change of unconscious neural priming in the cerebral language network. Proc Natl Acad Sci U S A 104:19643-19648.

Oldfield RC (1971) The assessment and analysis of handedness: the Edinburgh inventory. Neuropsychologia 9:97-113.

Pessiglione M, Schmidt L, Draganski B, Kalisch R, Lau H, Dolan RJ, Frith CD (2007) How the brain translates money into force: a neuroimaging study of subliminal motivation. Science 316:904-906.

Pessiglione M, Petrovic P, Daunizeau J, Palminteri S, Dolan RJ, Frith CD (2008) Subliminal instrumental conditioning demonstrated in the human brain. Neuron 59:561-567.

Pisella L, Gréa H, Tilikete C, Vighetto A, Desmurget M, Rode G, Boisson D, Rossetti Y (2000) An "automatic pilot" for the hand in human posterior parietal cortex: toward reinterpreting optic ataxia. Nat Neurosci 3:729-736.

Rees G, Kreiman G, Koch C (2002) Neural correlates of consciousness in humans. Nat Rev Neurosci 3:261-270.

Ridderinkhof KR, Ullsperger M, Crone EA, Nieuwenhuis S (2004) The role of the medial frontal cortex in cognitive control. Science 306:443-447.

Rubia K, Smith AB, Brammer MJ, Taylor E (2003) Right inferior prefrontal cortex mediates response inhibition while mesial prefrontal cortex is responsible for error detection. Neuroimage 20:351-358.

Schmajuk M, Liotti M, Busse L, Woldorff MG (2006) Electrophysiological activity underlying inhibitory control processes in normal adults. Neuropsychologia 44:384-395.

Simmonds DJ, Pekar JJ, Mostofsky SH (2008) Meta-analysis of go/no-go tasks demonstrating that fMRI activation associated with response inhibition is task-dependent. Neuropsychologia 46:224-232.

Stoerig P, Cowey A (1997) Blindsight in man and monkey. Brain 120 535-559.

Suhler CL, Churchland PS (2009) Control: conscious and otherwise. Trends Cogn Sci 13:341-347.

Sumner P, Husain M (2008) At the edge of consciousness: automatic motor activation and voluntary control. Neuroscientist 14:474-486.

Sumner P, Tsai PC, Yu K, Nachev P (2006) Attentional modulation of sensorimotor processes in the absence of perceptual awareness. Proc Natl Acad Sci U S A 103:10520-10525.

Sumner P, Nachev P, Morris P, Peters AM, Jackson SR, Kennard C, Husain M (2007) Human medial frontal cortex mediates unconscious inhibition of voluntary action. Neuron 54:697-711.

Thompson KG, Schall JD (1999) The detection of visual signals by macaque frontal eye field during masking. Nat Neurosci 2:283-288.

Umilta C (1988) The control operations of consciousness. In: Consciousness in contemporary science (Marcel AJ, Bisiach E, eds), pp 334-356. Oxford: Oxford UP.

van den Wildenberg WP, van Boxtel GJ, van der Molen MW, Bosch DA, Speelman JD, Brunia CH (2006) Stimulation of the subthalamic region facilitates the selection and inhibition of motor responses in Parkinson's disease. J Cogn Neurosci 18:626-636.

van Gaal S, Ridderinkhof KR, Fahrenfort JJ, Scholte HS, Lamme VA (2008) Frontal cortex mediates unconsciously triggered inhibitory control. J Neurosci 28:8053-8062.

van Gaal S, Ridderinkhof KR, van den Wildenberg WP, Lamme VA (2009) Dissociating consciousness from inhibitory control: evidence for unconsciously triggered inhibitory control in the stop-signal paradigm. J Exp Psychol Hum Percept Perform 35:1129-1139.

Verbruggen F, Logan GD (2008) Automatic and controlled response inhibition: associative learning in the go/no-go and stop-signal paradigms. J Exp Psychol Gen 137:649-672.

Wager TD, Sylvester CY, Lacey SC, Nee DE, Franklin M, Jonides J (2005) Common and unique components of response inhibition revealed by fMRI. Neuroimage 27:323-340.

Whalen PJ, Rauch SL, Etcoff NL, McInerney SC, Lee MB, Jenike MA (1998) Masked presentations of emotional facial expressions modulate amygdala activity without explicit knowledge. J Neurosci 18:411-418.

Woolrich MW, Ripley BD, Brady M, Smith SM (2001) Temporal autocorrelation in univariate linear modeling of FMRI data. Neuroimage 14: $1370-1386$

Worsley KJ (2001) Statistical analysis of activation images. In: Functional magnetic resonance imaging of the brain: methods for neuroscience (Matthews PM, Jezzard P, Smith SM, eds). London: Oxford UP.

Worsley KJ, Marrett S, Neelin P, Vandal AC, Friston KJ, Evans AC (1996) A unified statistical approach for determining significant signals in images of cerebral activation. Hum Brain Mapp 4:58-73.

Xue G, Aron AR, Poldrack RA (2008) Common neural substrates for inhibition of spoken and manual responses. Cereb Cortex 18:1923-1932. 